\title{
ON ORTHOGONALLY EXPONENTIAL FUNCTIONALS
}

\author{
JANUSZ BRZDȨK
}

Let $(X, \perp)$ be an orthogonality space and $g: X \rightarrow \mathbf{C}, g(X) \neq$ $\{0\}$, be an orthogonally exponential functional, hemicontinuous at the origin. We show that then one of the follwing two conditions is valid:

(i) There are unique linear functionals $a_{1}, a_{2}: X \rightarrow \mathbf{R}$ with

$$
g(x)=\exp \left(a_{1}(x)+i a_{2}(x)\right) \quad \text { for } x \in X
$$

(ii) there are a $\perp$-equivalent inner product $\langle\cdot, \cdot\rangle$ in $X, c \in \mathbf{C}$, and unique linear functionals $a_{1}, a_{2}: X \rightarrow \mathbf{R}$ such that

$$
g(x)=\exp \left(a_{1}(x)+i a_{2}(x)+c\|x\|^{2}\right) \quad \text { for } x \in X,
$$

where $\|x\|=\langle x, x\rangle$ for $x \in X$.

We also prove some auxiliary results concerning functions $f$ mapping a real linear (orthogonality) space $X$ into a commutative group $(G,+)$ and satisfying one of the following two conditions:

$$
\begin{aligned}
& f(x+y)+f(x-y)-2 f(x)-2 f(y) \in K \quad \text { for } \quad x, y \in X, \\
& f(x+y)-f(x)-f(y) \in K \quad \text { whenever } x \perp y,
\end{aligned}
$$

where $K$ is a subgroup of $G$.

\section{Introduction.}

We study the orthogonally exponential functionals on a linear space $X$, i.e. solutions of the conditional equation

$$
f(x+y)=f(x) f(y) \quad \text { whenever } x \perp y,
$$

where $\perp \subset X^{2}$ is a relation fulfilling some additional conditions. We obtain their description under some regularity conditions.

The results which we prove correspond to the outcomes in numerous papers concerning the orthogonally additive mapping (see e.g. [1, pp. 185-194], $[8],[9],[10],[12],[13],[14])$, i.e. solutions of the equation

$$
f(x+y)=f(x)+f(y) \quad \text { whenever } x \perp y,
$$


and to some results in [2], [3] and [5].

Let $\mathbf{N}, \mathbf{Z}, \mathbf{Q}, \mathbf{R}$ and $\mathbf{C}$ denote, as usual, the sets of all positive integers, integers, rationals, reals and complex numbers, respectively. Following [9] and [13] (cf. also [10]), we introduce the subsequent definition.

Definition 1.1. A pair $(X, \perp)$ is an orthogonality space provided $X$ is a real linear space with $\operatorname{dim} X \geq 2$ and $\perp \subset X^{2}$ is a relation such that

(01) $x \perp 0$ and $0 \perp x$ for every $x \in X$;

(02) if $x, y \in X \backslash\{0\}$ and $x \perp y$, then $x$ and $y$ are linearly independent;

(03) if $x, y \in X$ and $x \perp y$, then $a x \perp$ by for every $a, b \in \mathbf{R}$;

$\left(04^{\prime}\right)$ if $P$ is a 2-dimensional subspace of $X, x \in P$ and $a \in \mathbf{R}, a>0$, then there is $y \in P$ with $x \perp y$ and $x+y \perp a x-y$.

Actually our condition (04') is stronger than conditions (04) and (05) in [9]; however, this does not exclude any of the examples given in [9] (cf. $[13$, p. 36]). In the examples each of the following three relations makes a real linear space $X$ into an orthogonality space (see [9] and [13]).

Example A. The trivial orthogonality; i.e. defined by (01) and by the formula: For every $x, y \in X \backslash\{0\}, x \perp y$ if and only if $x$ and $y$ are linearly independent.

Example B. The ordinary orthogonality on a real inner product space $(X,\langle\cdot, \cdot\rangle)$; i.e. $x \perp y$ if and only if $\langle x, y\rangle=0$ (in the sequel we also call such a space a real inner product space).

Example C. The Birkhoff-James orthogonality on a normed linear space; i.e. $x \perp y$ if and only if $\|x+b y\| \geq\|x\|$ for every $b \in \mathbf{R}$.

In the case where $X$ is a real inner product space the orthogonally exponential functionals $f: X \rightarrow \mathbf{C}$ have been investigated in [2], [3] and [5].

\section{Auxiliary results.}

The results contained in this part are only auxiliary. However, to some extend, they correspond to or generalize certain outcomes in [2]-[7] and, therefore, they are in a more general settings than it is necessary for the proofs of our main theorems.

We begin with a definition and two lemmas.

Definition 2.1. (Cf. $[\mathbf{1 1}$, p. 596].) Let $X$ be a real linear space and $D \subset X$. We say that a point $x \in X$ is algebraically interior to $D$ provided, for every $y \in X$, there is $c \in \mathbf{R}, c>0$, such that $x+(-c, c) y:=\{x+d y$ : $d \in(-c, c)\} \subset D$. We say that $D$ is algebraically open if every point of $D$ is algebraically interior to $D$. 
Lemma 2.2. Let $X$ be a real linear space and $D \subset X$ be a set such that the origin is algebraically interior to $D$. Then the following two conditions are valid.

(i) Every additive functional $a: X \rightarrow \mathbf{R}$ bounded above or below on $D$ is linear.

(ii) Every symmetric biadditive functional $L: X^{2} \rightarrow \mathbf{R}$ such that $|L(x, x)|<$ $e$ for $x \in D$, with some $e>0$, satisfies

$$
L(c z, d z)=c d L(z, z) \quad \text { for } c, d \in \mathbf{R}, z \in X .
$$

If, moreover, $D$ is algebraically open, then $L$ is bilinear.

Proof. (i) Fix $x \in X \backslash\{0\}$ and define a function $a_{x}: \mathbf{R} \rightarrow \mathbf{R}$ by: $a_{x}(d)=a(d x)$ for $d \in \mathbf{R}$. Further, put $D_{x}=\{d \in \mathbf{R}: d x \in D\}$. Then $a_{x}$ is additive and bounded on $D_{x}$, and $0 \in \operatorname{int} D_{x}$. Thus (cf. [1], Corollary 2.1.5) there is $d_{x} \in \mathbf{R}$ with $a_{x}(d)=d_{x} d$ for $d \in \mathbf{R}$. This yields the assertion (i). by

(ii) Take $z \in X \backslash\{0\}$ and, for every $d \in \mathbf{R}$, define a function $h_{d}: \mathbf{R} \rightarrow \mathbf{R}$

$$
h_{d}(c)=L(c z, d z) \quad \text { for } c \in \mathbf{R} .
$$

Then $h_{d}$ is additive for every $d \in \mathbf{R}$. Further, according to the hypothesis on $D$, there is $d_{z}>0$ with $\left(-2 d_{z}, 2 d_{z}\right) z \subset D$. Thus, for every $c, d \in\left(-d_{z}, d_{z}\right)$, we have $c z+d z, c z-d z \in D$ and consequently

$$
\left|h_{d}(c)\right|=\frac{1}{4}|L(c z+d z, c z+d z)-L(c z-d z, c z-d z)|<e,
$$

which, in view of (i), means that for every $d \in\left(-d_{z}, d_{z}\right)$ there is $c_{d} \in \mathbf{R}$ with

$$
h_{d}(c)=c_{d} c \quad \text { for } c \in \mathbf{R} .
$$

Since

$$
h_{n d}(c)=L(c z, n d z)=n L(c z, d z)=n h_{d}(c) \quad \text { for every } c, d \in \mathbf{R}, n \in \mathbf{Z},
$$

this implies that $h_{d}$ has this form for every $d \in \mathbf{R}$. Whence, by the symmetry of $L$, the biadditive function $L_{z}: \mathbf{R}^{2} \rightarrow \mathbf{R}$, given by

$$
L_{z}(c, b)=L(c z, b z) \quad \text { for } \quad c, b \in \mathbf{R},
$$

is bilinear. This yields (3).

To complete the proof suppose that $D$ is algebraically open. Take $x, y \in$ $X$. There is $m \in \mathbf{N}$ with $2^{-m} y \in D$, which means that $2^{-m} y$ is algebraically interior to $D$. Thus there exists a nontrivial real interval $I$ such that, for every $c \in I, c 2^{-m} x+2^{-m} y \in D$ and consequently

$$
L(c x+y, c x+y)=2^{2 m} L\left(2^{-m}(c x+y), 2^{-m}(c x+y)\right)<2^{2 m} e .
$$


Now, using Propositions 11.1.1 and 11.1.4 in [1] we obtain the bilinearity of $L$. This ends the proof.

Lemma 2.3. Let $X$ be a real linear space, $(G,+)$ be a group (not necessarily commutative) without elements of order 2 (i.e. $2 x:=x+x \neq 0$ for every $x \in G \backslash\{0\})$, and $W$ be a subset of $X$ with $W \subset 2 W:=\{2 x: x \in W\}$ and

$$
X=\bigcup\left\{2^{n} W: n \in \mathbf{N}\right\} .
$$

Suppose that a function $f: W \rightarrow G$ satisfies

$$
f(x+y)+f(x-y)=2 f(x)+2 f(y)
$$

for every $x, y \in W$ with $x+y, x-y \in W$. Then there is exactly one quadratic function $q: X \rightarrow G$, i.e. satisfying the equation

$$
q(x+y)+q(x-y)=2 q(x)+2 q(y) \quad \text { for } x, y \in X,
$$

such that $f(x)=q(x)$ for $x \in W$.

Proof. According to (4), $0 \in W$. Setting in (5) first $x=y=0$ and next $x=y$ we obtain $f(0)=0$ and then

$$
f(2 x)=4 f(x) \quad \text { for } x \in W \text { with } 2 x \in W .
$$

Thus by induction, we get

$$
f\left(2^{n} x\right)=2^{2 n} f(x) \quad \text { for } n \in \mathbf{N}, x \in W \text { with } 2^{n} x \in W .
$$

Hence for every $m, n \in \mathbf{N} \cup\{0\}, m>n$, and $x \in X$ with $2^{-n} x \in W$,

$$
2^{2 m} f\left(2^{-m} x\right)=2^{2 n} 2^{2(m-n)} f\left(2^{-m} x\right)=2^{2 n} f\left(2^{-n} x\right) .
$$

This means that we can define a function $q: X \rightarrow G$ by the formula:

$$
q(x)=2^{2 n} f\left(2^{-n} x\right) \quad \text { for } x \in X,
$$

with some $n \in \mathbf{N}$ such that $2^{-n} x \in W$. It is easily seen that $q$ is an extension of $f$.

Now, we prove that $q$ is a solution of (Q). For this fix $z, w \in X$. Since $W \subset 2 W$, by (4) there is $n \in \mathbf{N}$ such that

$$
2^{-n} z, 2^{-n} w,-2^{-n} w, 2^{-n}(z+w), 2^{-n}(z-w), 2^{-n}(w-z) \in W .
$$

Further, putting in (5) $x=0$, we have

$$
f(-y)=f(y) \quad \text { for } y \in W \text { with }-y \in W .
$$


Thus

$$
\begin{aligned}
& f\left(2^{-n}(z+w)\right)+f\left(2^{-n}(z-w)\right)=2 f\left(2^{-n} z\right)+2 f\left(2^{-n} w\right) \\
& =2 f\left(2^{-n} z\right)+2 f\left(-2^{-n} w\right)=f\left(2^{-n}(z-w)\right)+f\left(2^{-n}(z+w)\right)
\end{aligned}
$$

and

$$
\begin{aligned}
& 2 f\left(2^{-n} z\right)+2 f\left(2^{-n} w\right)=f\left(2^{-n}(z+w)\right)+f\left(2^{-n}(z-w)\right) \\
& =f\left(2^{-n}(w+z)\right)+f\left(2^{-n}(w-z)\right)=2 f\left(2^{-n} w\right)+2 f\left(2^{-n} z\right) .
\end{aligned}
$$

Whence and by virtue of (5)

$$
\begin{aligned}
& q(z+w)+q(z-w)=2^{2 n} f\left(2^{-n}(z+w)\right)+2^{2 n} f\left(2^{-n}(z-w)\right) \\
& =2^{2 n}\left(f\left(2^{-n}(z+w)\right)+f\left(2^{-n}(z-w)\right)\right)=2^{2 n}\left(2 f\left(2^{-n} z\right)+2 f\left(2^{-n} w\right)\right) \\
& =2 q(z)+2 q(w) .
\end{aligned}
$$

It remains to show that $q$ is unique. So suppose that $r: X \rightarrow G$ is also a solution of (5) and $r(x)=f(x)$ for $x \in W$. Take $x \in X$. There is $n \in \mathbf{N}$ with $2^{-n} x \in W$. Thus

$$
r(x)=2^{2 n} r\left(2^{-n} x\right)=2^{2 n} f\left(2^{-n} x\right)=q(x) .
$$

This completes the proof.

In what follows, given a non-empty subset $U$ of a group $(G,+)$, we put $U^{(1)}=U$ and $U^{(n+1)}=U^{(n)}+U:=\left\{x+y: x \in U^{(n)}, y \in U\right\}$ for $n \in \mathbf{N}$.

The next proposition and two theorems correspond to the results contained in [4], [6] and [7].

Proposition 2.4. Let $X$ and $W$ be just the same as in Lemma 2.3. Suppose that $(G,+)$ is a commutative group without elements of order $2, K$ is a subgroup of $G, U \neq \varnothing$ is a subset of $G$ with $U=-U$ and $U^{(6)} \cap K=\{0\}$, and $f: X \rightarrow G$ is a function such that $f(W)-f(0) \subset K+U$ and

$$
f(x+y)+f(x-y)-2 f(y)-2 f(x) \in K \quad \text { for } x, y \in X .
$$

Then $2 f(0) \in K$ and there is a quadratic function $q: X \rightarrow G$ such that

$$
f(x)-q(x)-f(0) \in K \quad \text { for } x \in X .
$$

Furthermore, $q(W) \subset U$.

Proof. There are functions $u: W \rightarrow U$ and $k: W \rightarrow K$ such that $f(x)-$ $f(0)=k(x)+u(x)$ for $x \in W$. Further, for every $x, y \in W$ with $x+y, x-y \in$ 
$W$

$$
\begin{aligned}
& u(x+y)+u(x-y)-2 u(y)-2 u(x) \\
& =[f(x+y)+f(x-y)-2 f(x)-2 f(y)] \\
& \quad+2 f(0)-k(x+y)-k(x-y)+2 k(y)+2 k(x)
\end{aligned}
$$

and consequently

$$
u(x+y)+u(x-y)-2 u(y)-2 u(x) \in K \cap U^{(6)}=\{0\},
$$

because, by (6), $2 f(0) \in K$. Hence, by Lemma 2.3 , there is a quadratic function $q: X \rightarrow G$ being an extension of $u$, which means that $q(W) \subset U$ and

$$
f(x)-q(x)-f(0) \in K \quad \text { for } \quad x \in W .
$$

Setting in (6) $x=y$ we get by induction

$$
f\left(2^{n} x\right)-2^{2 n} f(x)+f(0) \in K \quad \text { for } n \in \mathbf{N}, x \in X .
$$

Take $x \in X$. There is $n \in \mathbf{N}$ with $2^{-n} x \in W$. Thus (8) and (9) imply that

$$
\begin{aligned}
f(x)-q(x)-f(0)= & {\left[f(x)-2^{2 n} f\left(2^{-n} x\right)\right] } \\
& +2^{2 n}\left[f\left(2^{-n} x\right)-q\left(2^{-n} x\right)\right]-f(0) \in K \quad \text { for } \quad x \in X
\end{aligned}
$$

(because $2 f(0) \in K$ ). This completes the proof.

Now, using Proposition 2.4 we can prove the subsequent two theorems; but to formulate them we need one more definition.

Definition 2.5. Let $(G,+)$ be a topological group. We say that a subgroup $K$ of $G$ is discrete provided there is a neighbourhood $U \subset G$ of 0 with $K \cap U=\{0\}$.

Theorem 2.6. Let $X$ be a real topological linear space, $(G,+)$ be a commutative topological group without elements of order $2, K$ be a discrete subgroup of $G$, and $f: X \rightarrow G$ be a continuous at the origin function satisfying (6). Then $2 f(0) \in K$ and there is a unique quadratic function $q: X \rightarrow G$, continuous at the origin, such that (7) holds.

Proof. Let $U, V \subset G$ be neighbourhoods of 0 such that $U \cap K=\{0\}, V=$ $-V$, and $V^{(6)} \subset U$. Since $f$ is continuous at the origin, there is a balanced neighbourhood $W \subset X$ of the origin with $f(W) \subset f(0)+V$, which yields

$$
f(W)-f(0) \subset V \text {. }
$$


Thus, by Proposition 2.4, $2 f(0) \in K$ and there is a quadratic function $q: X \rightarrow G$ such that $(7)$ holds and $q(W) \subset V$, whence

$$
f(x)-q(x)-f(0) \in K \cap(V-V) \subset K \cap U=\{0\} \quad \text { for } \quad x \in W .
$$

Hence $q(x)=f(x)-f(0)$ for $x \in W$, which means that $q$ is continuous at the origin.

To complete suppose that $r: X \rightarrow G$ is also a quadratic and continuous at the origin function with $f(x)-r(x)-f(0) \in K$ for $x \in X$. Then $q(x)-r(x) \in$ $K$ for $x \in X$. Let $U, V \subset G$ be neighbourhoods of 0 such that $K \cap U=\{0\}$ and $V-V \subset U$. There is a neighbourhood $W \subset X$ of the origin with $q(W), r(W) \subset V$, which implies

$$
q(x)-r(x) \in K \cap(V-V) \subset K \cap U=\{0\} \quad \text { for } x \in W .
$$

Further, for every $x \in X$, there is $n(x) \in \mathbf{N}$ with $2^{-n(x)} x \in W$. Hence

$$
q(x)=2^{2 n(x)} q\left(2^{-n(x)} x\right)=2^{2 n(x)} r\left(2^{-n(x)} x\right)=r(x) \quad \text { for } x \in X .
$$

This ends the proof

Theorem 2.7. Let $X$ be a real linear space and $f: X \rightarrow \mathbf{R}$ be a function satisfying (6) with $K=\mathbf{Z}$. Suppose that there is a set $D \subset X$ such that the origin is algebraically interior to $D$ and

$$
f(D)-f(0) \subset \mathbf{Z}+\left(-\frac{1}{5}, \frac{1}{5}\right)
$$

Then $2 f(0) \in \mathbf{Z}$ and there exists exactly one biadditive symmetric function $L: X^{2} \rightarrow \mathbf{R}$ such that

$$
f(x)-L(x, x)-f(0) \in \mathbf{Z} \quad \text { for } x \in X .
$$

Moreover, (3) holds.

Proof. For every $x \in X \backslash\{0\}$ there is $d_{x} \in \mathbf{R}, d_{x}>0$, with $\left(-d_{x}, d_{x}\right) x \subset D$. Put

$$
W=\frac{1}{2}\left[\bigcup\left\{\left(-d_{x}, d_{x}\right) x: x \in X \backslash\{0\}\right\}\right] .
$$

It is easily seen that $W \subset 2 W \subset D$ and (4) is valid.

It results from (6) that $2 f(0) \in \mathbf{Z}$ and

$$
4 f\left(\frac{1}{2} y\right)-f(0) \in f(y)+\mathbf{Z} \quad \text { for } y \in X
$$

which implies $4 f(W)-4 f(0) \subset f(2 W)-f(0)+\mathbf{Z} \subset \mathbf{Z}+\left(-\frac{1}{5}, \frac{1}{5}\right)$. 
Next, by (10),

$4 f(D)-4 f(0) \subset 4 \mathbf{Z}+\left(-\frac{4}{5}, \frac{4}{5}\right)$.

Thus

$$
4 f(W)-4 f(0) \subset\left[\mathbf{Z}+\left(-\frac{1}{5}, \frac{1}{5}\right)\right] \cap\left[4 \mathbf{Z}+\left(-\frac{4}{5}, \frac{4}{5}\right)\right]=4 \mathbf{Z}+\left(-\frac{1}{5}, \frac{1}{5}\right) .
$$

Hence

$$
f(W)-f(0) \subset \mathbf{Z}+\left(-\frac{1}{20}, \frac{1}{20}\right)
$$

and consequently, on account of Proposition 2.4, there is a quadratic function $q: X \rightarrow \mathbf{R}$ such that (7) holds and

$$
q(W) \subset\left(-\frac{1}{20}, \frac{1}{20}\right)
$$

Now, using Proposition 11.1.1 in [1], we derive that there is a biadditive and symmetric function $L: X^{2} \rightarrow \mathbf{R}$ with $L(x, x)=q(x)$ for $x \in X$. This yields (11) and

$$
|L(x, x)|<\frac{1}{20} \quad \text { for } \quad x \in W,
$$

which, according to Lemma 2.2(ii), implies (3).

It remains to show that $L$ is unique. So, suppose that $S: X^{2} \rightarrow \mathbf{R}$ is also a biadditive and symmetric function with $f(x)-S(x, x)-f(0) \in \mathbf{Z}$ for $x \in X$. Then

$$
L(x, x)-S(x, x) \in \mathbf{Z} \quad \text { for } x \in X .
$$

Next, for every $x \in X$, there is $n \in \mathbf{N}$ with

$$
2^{-2 n}|L(x, x)-S(x, x)|<1,
$$

which implies that

$$
L\left(2^{-n} x, 2^{-n} x\right)-S\left(2^{-n} x, 2^{-n} x\right)=0
$$

and consequently

$$
L(x, x)=2^{2 n} L\left(2^{-n} x, 2^{-n} x\right)=2^{2 n} S\left(2^{-n} x, 2^{-n} x\right)=S(x, x) .
$$


Whence, for every $x, y \in X$

$$
\begin{aligned}
L(x, y) & =L\left(\frac{1}{2}(x+y), \frac{1}{2}(x+y)\right)-L\left(\frac{1}{2}(x-y), \frac{1}{2}(x-y)\right) \\
& =S\left(\frac{1}{2}(x+y), \frac{1}{2}(x+y)\right)-S\left(\frac{1}{2}(x-y), \frac{1}{2}(x-y)\right)=S(x, y) .
\end{aligned}
$$

This completes the proof.

Remark. The function $L$ in Theorem 2.7 need not to be bilinear. In fact, let $S: X^{2} \rightarrow \mathbf{R}$ be a symmetric and biadditive function satisfying

$$
S(c x, c x)=c^{2} S(x, x) \quad \text { for } c \in \mathbf{R}, x \in X,
$$

which is not bilinear (see [1], Proposition 11.1.6). Put $f(x)=S(x, x)$ for $x \in X$. Then $f(0)=0$ and $f$ satisfies (6) with $K=\{0\}$ (cf. [1], Proposition 11.1.1). Further, for every $x \in X \backslash\{0\}$ there is $c_{x} \in \mathbf{R}, c_{x}>0$, with

$$
f\left(\left(-c_{x}, c_{x}\right) x\right)=\left[0, c_{x}^{2}\right) f(x) \subset\left(-\frac{1}{5}, \frac{1}{5}\right)
$$

and consequently (10) holds with

$$
D=\bigcup\left\{\left(-c_{x}, c_{x}\right) x: x \in X\right\} .
$$

It is easily seen that the origin is algebraically interior to $D$.

We need also the following proposition.

Proposition 2.8. Let $X$ be a real linear space, $D$ be a subset of $X$ such that the origin is algebraically interior to $D, e \in \mathbf{R}, 0<2 e<1$, and $f: X \rightarrow \mathbf{R}$ be a function with

$$
f(x+y)-f(x)-f(y) \in \mathbf{Z} \quad \text { for } x, y \in X
$$

and $f(D) \subset(-e, e)+\mathbf{Z}$. Then there exists a unique linear functional $a$ : $X \rightarrow \mathbf{R}$ with

$$
f(x)-a(x) \in \mathbf{Z} \quad \text { for } x \in X \text {. }
$$

Proof. For every $x \in X \backslash\{0\}$ put $D_{x}=\{d \in \mathbf{R}: d x \in D\}$ and define a function $f_{x}: \mathbf{R} \rightarrow \mathbf{R}$ by $f_{x}(d)=f(d x)$ for $d \in \mathbf{R}$. Then $f_{x}$ satisfies (12) (with $X=\mathbf{R}$ ) and $f_{x}\left(D_{x}\right) \subset(-e, e)+\mathbf{Z}$. Thus, in view of Corollary 1 in [7], for every $x \in X \backslash\{0\}$ there is $c_{x} \in \mathbf{R}$ such that

$$
f_{x}(d)-c_{x} d \in \mathbf{Z} \quad \text { for } d \in \mathbf{R} .
$$


Suppose that $c_{x}^{\prime} \in \mathbf{R}$ also satisfies $f_{x}(d)-c_{x}^{\prime} d \in \mathbf{Z}$ for $d \in \mathbf{R}$. Then $\left(c_{x}-c_{x}^{\prime}\right) d \in$ $\mathbf{Z}$ for $d \in \mathbf{R}$, which yields $c_{x}=c_{x}^{\prime}$.

Since $c_{x}$ is unique, we may define a functional $a: X \rightarrow \mathbf{R}$ by $a(x)=c_{x}$ for $x \in X \backslash\{0\}$ and $a(0)=0$. Then

$$
f(x)-a(x)=f_{x}(1)-c_{x} \in \mathbf{Z} \quad \text { for } x \in X \backslash\{0\} .
$$

Further, by (12), $f(0) \in \mathbf{Z}$. Thus it remains to show that $a$ is linear and unique.

To this end fix $b \in \mathbf{R}$ and $x, y \in X$. Then, for every $d \in \mathbf{R}$,

$$
\begin{aligned}
d\left(c_{b x+y}-b c_{x}-c_{y}\right)= & c_{b x+y} d-f_{b x+y}(d)-\left(c_{x} d b-f_{x}(d b)\right) \\
& -\left(c_{y} d-f_{y}(d)\right)+f(d(b x+y))-f(d b x)-f(d y) \in \mathbf{Z} .
\end{aligned}
$$

Hence $a(b x+y)=b a(x)+a(y)$.

Finally, suppose that $a^{\prime}: X \rightarrow \mathbf{R}$ is linear and $f(x)-a^{\prime}(x) \in \mathbf{Z}$ for $x \in X$. Then, for every $d \in \mathbf{R}, x \in X, d\left(a(x)-a^{\prime}(x)\right)=a(d x)-a^{\prime}(d x) \in \mathbf{Z}$, which means that $a=a^{\prime}$. This completes the proof.

Now, we are in a position to prove the following two theorems which generalize, to some extent, Corollary 1 in [2] and Theorem in [5].

Theorem 2.9. Let $X$ be an orthogonality space endowed with a linear topology (i.e. one which makes $X$ into a real linear topological space), $(G,+)$ be a commutative topological group without elements of order 2 , and $K$ be a discrete subgroup of $G$. Then a continuous at the origin function $f: X \rightarrow G$ satisfies

$$
f(x+y)-f(x)-f(y) \in K \quad \text { whenever } x \perp y
$$

if and only if there exist a unique continuous additive function $a: X \rightarrow G$ and a unique continuous at the origin quadratic and orthogonallly additive function $q: X \rightarrow G$ with

$$
f(x)-a(x)-q(x) \in K \quad \text { for } x \in X .
$$

Proof. First suppose that $f$ satisfies (13). Define functions $f_{1}, f_{2}: X \rightarrow G$ and $F_{1}, F_{2}: X \rightarrow G / K$ by the formulae

$$
\begin{array}{ll}
f_{1}(x)=f(x)-f(-x) & \text { for } x \in X, \\
f_{2}(x)=f(x)+f(-x) & \text { for } x \in X,
\end{array}
$$

and $F_{i}=p \circ f_{i}$ for $i=1,2$, where $p$ is the natural projection of $G$ onto the factor group $G / K$. Then $f_{1}$ and $f_{2}$ are continuous at the origin, $f_{i}(x) \in F_{i}(x)$ 
for $x \in X, i=1,2, F_{1}$ is odd, $F_{2}$ is even, and $F_{1}, F_{2}$ are orthogonally additive. Thus, according to Theorems 5 and 6 in [13], $F_{1}$ is additive and $F_{2}$ is quadratic, which means that $f_{1}$ satisfies (12) (with $\mathbf{Z}$ replaced by $K)$ and $f_{2}$ satisfies (6). Moreover, by (13), $f(0) \in K$ and consequently $f_{2}(0)=2 f(0) \in K$. Hence, by Theorem 3 in [4] and our Theorem 2.6, there are a continuous additive function $a_{0}: X \rightarrow G$ and a quadratic function $q_{0}: X \rightarrow G$, continuous at the origin, such that

$$
f_{1}(x)-a_{0}(x), f_{2}(x)-q_{0}(x) \in K \quad \text { for } x \in X .
$$

Since $f_{2}$ is a solution of (13), (15) implies that so is $q_{0}$.

Let $k: X \rightarrow K$ be the function given by

$$
k(x)=2 f(x)-a_{0}(x)-q_{0}(x) \quad \text { for } x \in X .
$$

Then, for every $x, y \in X$ with $x \perp y$,

$$
\begin{aligned}
k(x+y)-k(x)-k(y)= & 2[f(x+y)-f(x)-f(y)] \\
& -4\left[q_{0}\left(\frac{1}{2}(x+y)\right)-q_{0}\left(\frac{1}{2} x\right)-q_{0}\left(\frac{1}{2} y\right)\right] \in 2 K .
\end{aligned}
$$

We have also

$$
k(x)+k(-x)=2\left[f_{2}(x)-q_{0}(x)\right] \in 2 K \quad \text { for } x \in X .
$$

Thus the function $k_{0}: X \rightarrow K / 2 K$, defined by:

$$
k_{0}(x)=k(x)+2 K \quad \text { for } x \in X,
$$

is odd and orthogonally additive, which, in view of Theorem 5 in [13], means that it is additive. Hence

$$
k_{0}(x)=2 k_{0}\left(\frac{1}{2} x\right)=0 \quad \text { for } x \in X
$$

and consequently $k(X) \subset 2 K$.

Thus we have shown that

$$
2\left[f(x)-a_{0}\left(\frac{1}{2} x\right)-2 q_{0}\left(\frac{1}{2} x\right)\right]=k(x) \in 2 K \quad \text { for } \quad x \in X .
$$

Put $a(x)=a_{0}\left(\frac{1}{2} x\right)$ and $q(x)=2 q_{0}\left(\frac{1}{2} x\right)$ for $x \in X$. It is easily seen that (14) holds, $a$ is continuous and additive, and $q$ is continuous at the origin and quadratic.

It remains to prove that $q$ is orthogonally additive and $q$ and $a$ are unique. Let $U, V \subset G$ be neighbourhoods of 0 such that $K \cap U=\{0\}, V=-V$, and 
$V^{(3)} \subset U$. Since $q$ is continuous at the origin, there is a neighbourhood $W \subset X$ of the origin with $q(W) \subset V$. Take $x, y \in X$ with $x \perp y$. There exists $n \in \mathbf{N}$ with $2^{-n} x, 2^{-n} y, 2^{-n}(x+y) \in W$. Thus, by (13) and (14),

$$
q\left(2^{-n}(x+y)\right)-q\left(2^{-n} x\right)-q\left(2^{-n} y\right) \in K \cap V^{(3)} \subset K \cap U=\{0\},
$$

which yields

$$
q(x+y)-q(x)-q(y)=2^{2 n}\left(q\left(2^{-n}(x+y)\right)-q\left(2^{-n} x\right)-q\left(2^{-n} y\right)\right)=0 .
$$

To complete the "only if" part of the proof suppose that $b, r: X \rightarrow G$ are also additive and quadratic functions, respectively, which are continuous at the origin and satisfy

$$
f(x)-b(x)-r(x) \in K \quad \text { for } \quad x \in X .
$$

Then

$$
a(x)-b(x)+q(x)-r(x) \in K \quad \text { for } \quad x \in X
$$

and consequently, for every $x \in X$,

$$
\begin{aligned}
a(2 x)-b(2 x)= & {[a(x)-b(x)+q(x)-r(x)] } \\
& -[a(-x)-b(-x)+q(-x)-r(-x)] \in K .
\end{aligned}
$$

Further, there is a neighbourhood $W \subset X$ of the origin such that $a(W), b(W) \subset$ $V$. Take $x \in X$. There exists $n \in \mathbf{N}$ with $2^{-n} x \in W$, which means that

$$
a\left(2^{-n} x\right)-b\left(2^{-n} x\right) \in K \cap V^{(2)} \subset K \cap U=\{0\},
$$

whence $a(x)=2^{n} a\left(2^{-n} x\right)=2^{n} b\left(2^{-n} x\right)=b(x)$.

So we have shown that $a=b$. Thus, by (16),

$$
q(x)-r(x) \in K \quad \text { for } x \in X \text {. }
$$

Reasoning analogously as for $a$ and $b$, we get $q=r$, which completes the "only if" part of the proof. Since the converse is easy to check, this ends the proof.

We can reformulate Theorem 2.9 in the following way.

Corollary 2.10. Let $X, G$, and $K$ be the same as in Theorem 2.9. Then a function $f: X \rightarrow G$, continuous at the origin, satisfies (13) if and only if 
there exist a unique continuous additive function $a: X \rightarrow G$ and a unique biadditive symmetric function $L: X^{2} \rightarrow G$, continuous at $(0,0)$, with

$$
f(x)-a(x)-L(x, x) \in K \quad \text { for } x \in X
$$

and

$$
L(x, y)=0 \quad \text { for } x \perp y .
$$

Proof. Arguing as in the proof of Proposition 11.1.1 in [1] (p. 166) one can show that $q: X \rightarrow G$ is a quadratic function if and only if there is a unique biadditive symmetric function $L: X^{2} \rightarrow G$ with $q(x)=L(x, x)$ for $x \in X$. There is only one difference. Here we define $L$ by:

$$
L(x, y)=q\left(\frac{1}{2}(x+y)\right)-q\left(\frac{1}{2}(x-y)\right) \quad \text { for } x, y \in X .
$$

Now, it is enough to use Theorem 2.9. The uniqueness of $L$ results from the uniqueness of $q$ in Theorem 2.9.

It remains to show that $L$ is continuous at $(0,0)$. To this end fix neighbourhoods $T, U \subset G$ of 0 with $T-T \subset U$. There are neighbourhoods $W, V \subset X$ of the origin such that $q(V) \subset T, W=-W$, and $W+W \subset 2 V$. Thus, by (19),

$$
L(W \times W) \subset q\left(\frac{1}{2}(W+W)\right)-q\left(\frac{1}{2}(W-W)\right) \subset q(V)-q(V) \subset T-T \subset U .
$$

This completes the proof.

Theorem 2.11. Let $X$ be an orthogonality space, $D$ be a subset of $X$ such that the origin is algebraically interior to $D, K$ be either $\mathbf{Z}$ or $\{0\}$ (here $0 \in \mathbf{R}$ ), and $f: X \rightarrow \mathbf{R}$ be a function with

$$
f(D) \subset\left(-\frac{1}{10}, \frac{1}{10}\right)+K
$$

Then $f$ satisfies (13) if and only if there exist a unique linear functional $a: X \rightarrow \mathbf{R}$ and a unique bilinear symmetric functional $L: X^{2} \rightarrow \mathbf{R}$ such that (17) and (18) are valid.

Proof. Suppose that $f$ satisfies (13). In the same way as in proof of Theorem 2.9 (with $G=\mathbf{R}$ ) we define functions $f_{1}, f_{2}: X \rightarrow \mathbf{R}$ and show that $f_{1}$ satisfies (12) (with $\mathbf{Z}$ replaced by $K$ ) and $f_{2}$ satisfies (6). Further, we have $f_{2}(0)=2 f(0) \in K$ and

$$
f_{1}(D), f_{2}(D) \subset K+\left(-\frac{1}{5}, \frac{1}{5}\right) .
$$


Thus, according to Proposition 2.8 and Theorem 2.7, there are a linear functional $a_{0}: X \rightarrow \mathbf{R}$ and a biadditive symmetric functional $L_{0}: X^{2} \rightarrow \mathbf{R}$ such that (3) holds (with $L=L_{0}$ ) and

$$
f_{1}(x)-a_{0}(x), f_{2}(x)-L_{0}(x, x) \in K \quad \text { for } x \in X .
$$

(Actually, in the case $K=\{0\}$ we cannot simply apply the above results. However, then $f_{1}$ is additive and $f_{2}$ is quadratic. Next, we use Proposition 11.1.1 in [1] and Lemma 2.2(ii).)

Again in analogous way as in the proof of Theorem 2.9, we prove that

$$
f(x)-a_{0}\left(\frac{1}{2} x\right)-2 L_{0}\left(\frac{1}{2} x, \frac{1}{2} x\right) \in K \quad \text { for } x \in X
$$

(notice that the function $q_{0}(x):=L_{0}(x, x)$ for $x \in X$ is quadratic). Putting

$$
a(x)=a_{0}\left(\frac{1}{2} x\right) \quad \text { and } \quad L(x, y)=2 L_{0}\left(\frac{1}{2} x, \frac{1}{2} y\right) \quad \text { for } x, y \in X
$$

we get (17) and (3).

Take $x, y \in X$ with $x \perp y$. Then $s x \perp s y$ for every $s \in \mathbf{Q}$. Thus, by (13) and $(17), 2 s^{2} L(x, y)=2 L(s x, s y) \in K$ for $s \in \mathbf{Q}$, which yields $L(x, y)=0$. Hence (18) holds, too. This means that the function $q: X \rightarrow \mathbf{R}$, defined by $q(x)=L(x, x)$ for $x \in X$, is orthogonally additive. Further, in view of (3), $q$ is hemicontinuous (see [9], p. 427). Consequently Theorem 2.2 in [9] and Proposition 11.1 .1 in [1] imply that $L$ is bilinear.

To complete the "only if" part of the proof it remains to show the uniqueness of $a$ and $L$. So suppose that $b: X \rightarrow \mathbf{R}$ and $S: X^{2} \rightarrow \mathbf{R}$ are also functionals, additive and biadditive, respectively, such that (17) and (18) are valid with $a$ replaced by $b$ and $L$ replaced by $S$. Then, in the same way as in the proof of Theorem 2.9, we show that $a(x)-b(x) \in K$ for $x \in X$. Next, analogously as in the proof of the uniqueness of $L$ of Theorem 2.7 we show that $a=b$. Repeating that for $L$ and $S$ we obtain also $L=S$.

Since the converse is easy to check, this completes the proof.

The next corollary reformulates Theorem 2.11 in a similar way as in Theorem 2.2 in [9]. For this we need two more definitions.

Definition 2.12. (Cf. [9], p. 427.) Let $X$ be a real linear space. We say that a function $f: X \rightarrow \mathbf{R}$ is hemicontinuous at the origin provided, for every $x \in X$, the function $f_{x}: \mathbf{R} \rightarrow \mathbf{R}$, given by

$$
f_{x}(a)=f(a x) \quad \text { for } a \in \mathbf{R},
$$

is continuous at 0 . 
Definition 2.13. Let $(X, \perp)$ be an orthogonality space and $\langle\cdot, \cdot\rangle$ be an inner product in $X$. We say that the inner product is $\perp$-equivalent provided for every $x, y \in X$

$$
x \perp y \quad \text { if and only if }\langle x, y\rangle=0 .
$$

Corollary 2.14. Let $(X, \perp)$ be an orthogonality space and $f: X \rightarrow \mathbf{R}$ be a hemicontinuous at the origin function. Then $f$ satisfies (13), with $K$ being either $\mathbf{Z}$ or $\{0\}$ (here $0 \in \mathbf{R}$ ), if and only if one of the following two conditions is valid:

(i) There is a linear functional $a: X \rightarrow \mathbf{R}$ with $f(x)-a(x) \in K$ for $x \in X$;

(ii) there exist $a \perp$-equivalent inner product $\langle\cdot, \cdot\rangle$ in $X$, a linear functional $a: X \rightarrow \mathbf{R}$, and $c \in\{-1,1\}$ such that $f(x)-a(x)-c\|x\|^{2} \in K$ for $x \in X$ (with $\|x\|^{2}=\langle x, x\rangle$ for $\left.x \in X\right)$.

Proof. The "if" part of the proof is trivial. So suppose that $f$ satisfies (13) and (i) does not hold. Since $f$ is hemicontinuous at the origin, for every $x \in X \backslash\{0\}$ there is $d_{x} \in \mathbf{R}, d_{x}>0$, such that

$$
f\left(\left(-d_{x}, d_{x}\right) x\right) \subset f(0)+\left(-\frac{1}{10}, \frac{1}{10}\right) .
$$

Put

$$
D=\bigcup\left\{\left(-d_{x}, d_{x}\right) x: x \in X \backslash\{0\}\right\} .
$$

Then the origin is algebraically interior to $D$. Further, by (13), $f(0) \in K$, which means that

$$
f(D) \subset K+\left(-\frac{1}{10}, \frac{1}{10}\right)
$$

Hence, in view of Theorem 2.11, there exist a linear functional $a: X \rightarrow \mathbf{R}$ and a nontrivial bilinear symmetric function $L: X^{2} \rightarrow \mathbf{R}$ such that (17) and (18) are valid. Using Theorem 2.2 in [9] for the function $q: X^{2} \rightarrow \mathbf{R}$, given by: $q(x)=L(x, x)$ for $x \in X$, we obtain (ii). This completes the proof.

\section{Orthogonally exponential functionals.}

In this part we prove a next auxiliary proposition; this time concerning orthogonally exponential functionals. Let us start with a lemma. 
Lemma 3.1. Let $(X, \perp)$ be an orthogonality space and $f: X \rightarrow \mathbf{C}$ be a solution of (1). Then the following two statements are valid:

(i) If $z, w \in X, f(z) \neq 0, z \perp w$ or $w \perp z$, and $z+w \perp z-w$, then

$$
f\left(\frac{1}{2} w\right) f\left(-\frac{1}{2} w\right) \neq 0
$$

(ii) if $z \in X$ and $f(z)=0$, then $f(a z)=0$ for every $a \in \mathbf{R}, a>0$.

Proof. (i) Take $z, w \in X$ with $f(z) \neq 0, z \perp w$, and $z+w \perp z-w$. Then, by $(03)$,

$$
\frac{1}{2}(z+w) \perp \frac{1}{2}(z-w), \quad \frac{1}{2} z \perp \frac{1}{2} w \quad \text { and } \quad \frac{1}{2} z \perp-\frac{1}{2} w .
$$

Thus, according to (1),

$$
0 \neq f(z)=f\left(\frac{1}{2}(z+w)\right) f\left(\frac{1}{2}(z-w)\right)=f\left(\frac{1}{2} z\right)^{2} f\left(\frac{1}{2} w\right) f\left(-\frac{1}{2} w\right) .
$$

Since the case $w \perp z$ is analogous, this completes the proof of (i).

(ii) First we show that

$$
f(a z)=0 \quad \text { for } a>1 \text { and } z \in X \text { with } f(z)=0 .
$$

So fix $a>1$ and $z \in X$ with $f(z)=0$. On account of $\left(04^{\prime}\right)$ there is $y \in X$ with $z \perp y$ and $z+y \perp(a-1) z-y$. Thus

$$
f(a z)=f(z+y+(a-1) z-y)=f(z) f(y) f((a-1) z-y)=0,
$$

which means that (21) holds.

Now, we prove (ii). Let $z \in X, f(z)=0$, and $b:=\inf \{c \in \mathbf{R}: c>0$ and $f(c z)=0\}$. According to (21) it suffices to show that $b=0$. For the proof by contradiction suppose that $b>0$. Take $c>b$ with $c<2 b$ and set $v=c z$. Then

$$
f\left(\frac{1}{2} v\right) f\left(\frac{1}{4} v\right) \neq 0
$$

and, by (21), $f(v)=0$. Next, let $y \in X, v \perp y$, and $v+y \perp v-y$. According to $(22),(03)$, and (i)

$$
f\left(\frac{1}{4} y\right) f\left(-\frac{1}{4} y\right) \neq 0 .
$$

Since, by (03), $y+v \perp y-v,(23)$ and (i) imply

$$
f\left(-\frac{1}{8} v\right) \neq 0
$$


Further,

$$
0=f(v)=f\left(\frac{1}{2}(v+y)\right) f\left(\frac{1}{2}(v-y)\right)=f\left(\frac{1}{2} v\right)^{2} f\left(\frac{1}{2} y\right) f\left(-\frac{1}{2} y\right),
$$

which, on account of (22), means that

$$
\begin{aligned}
0 & =f\left(\frac{1}{2} y\right) f\left(-\frac{1}{2} y\right) \\
& =f\left(\frac{1}{4}(v+y)\right) f\left(\frac{1}{4}(y-v)\right) f\left(\frac{1}{4}(v-y)\right) f\left(-\frac{1}{4}(v+y)\right) \\
& =f\left(\frac{1}{4} v\right)^{2} f\left(-\frac{1}{4} v\right)^{2} f\left(\frac{1}{4} y\right)^{2} f\left(-\frac{1}{4} y\right)^{2} .
\end{aligned}
$$

Consequently (22) and (23) yield

$$
\begin{aligned}
0 & =f\left(-\frac{1}{4} v\right)=f\left(-\frac{1}{8}(v+y)\right) f\left(\frac{1}{8}(y-v)\right) \\
& =f\left(-\frac{1}{8} v\right)^{2} f\left(-\frac{1}{8} y\right) f\left(\frac{1}{8} y\right) .
\end{aligned}
$$

Since, on account of (22) and (i),

$$
f\left(-\frac{1}{8} y\right) f\left(\frac{1}{8} y\right) \neq 0
$$

this contradicts (24).

Proposition 3.2. Let $(X, \perp)$ and $f$ be the same as in Lemma 3.1. Then either $f(x)=0$ for $x \in X \backslash\{0\}$ or $f(x) \neq 0$ for $x \in X$.

Proof. Suppose that there is $x \in X$ with $f(x)=0$. If $x=0$, then for every $y \in X$

$$
f(y)=f(y+0)=f(y) f(0)=0 .
$$

So, it remains to consider the case $x \neq 0$.

Take $z \in X \backslash \mathbf{R} x$. Let $P$ be a subspace of $X$ generated by $x$ and $z$. There is $y \in P \backslash\{0\}$ with $x \perp y$ and $x+y \perp x-y$, which, by (03), implies

$$
\begin{aligned}
f(2 y) & =f(x+y) f(y-x)=f(x) f(y)^{2} f(-x)=0, \\
f(-2 y) & =f(x-y) f(-y-x)=f(x) f(-y)^{2} f(-x)=0, \\
f(-2 x) & =f(-x-y) f(y-x)=f(-x)^{2} f(y) f(-y) .
\end{aligned}
$$

Thus, according to Lemma 3.1(ii), $f(-y)=0=f(y)$ and consequently $f(-x)=0$. Since, in view of (02), there exist $a, b \in \mathbf{R}$ with $z=a x+b y$, Lemma 3.1(ii) yields $f(z)=f(a x) f(b y)=0$. This completes the proof. 


\section{Main theorems.}

Now, we are in a position to prove our main results.

Theorem 4.1. Let $(X, \perp)$ be an orthogonality space and $g: X \rightarrow \mathbf{C}$, $g(X) \neq\{0\}$, be a solution of (1), hemicontinuous at the origin. Then there exist $c \in \mathbf{C}$, unique linear functionals $a_{1}, a_{2}: X \rightarrow \mathbf{R}$, and a symmetric bilinear functional $L: X^{2} \rightarrow \mathbf{R}$, unique up to a multiplicative constant, such that (18) holds and

$$
g(x)=\exp \left(a_{1}(x)+i a_{2}(x)+c L(x, x)\right) \quad \text { for } x \in X
$$

If, moreover, $X$ is a real linear topological space and $g$ is continuous at the origin, then $a_{1}$ and $a_{2}$ are continuous and $L$ is continuous at $(0,0)$.

Proof. Since $g$ is hemicontinuous at $0, g(x) \neq 0$ for some $x \in X \backslash\{0\}$. Thus, in view of Proposition 3.2, $0 \notin g(X)$. Put $S=\{z \in \mathbf{C}:|z|=1\}$ and define functions $f: X \rightarrow \mathbf{R}, h: X \rightarrow S$, and $t:\left[-\frac{1}{2}, \frac{1}{2}\right) \rightarrow S$ by the formulae

$$
\begin{array}{ll}
f(x)=\log |g(x)| & \text { for } x \in X, \\
h(x)=\frac{g(x)}{|g(x)|} & \text { for } \quad x \in X, \\
t(d)=\exp (2 \pi i d) & \text { for } d \in\left[-\frac{1}{2}, \frac{1}{2}\right) .
\end{array}
$$

Then $g(x)=\exp \left(f(x)+2 \pi i t^{-1}(h(x))\right)$ for $x \in X$. Further, $f$ and $h_{0}:=$ $t^{-1} \circ h$ satisfy (13) (with $K=\{0\}$ and $K=\mathbf{Z}$, respectively) and they are hemicontinuous at the origin. Arguing in the same way as in the proof of Corollary 2.14 it easy to show that the assumptions of Theorem 2.11 are fulfilled and consequently there are linear functionals $a_{1}, a_{0}: X \rightarrow \mathbf{R}$ and symmetric bilinear functionals $L_{1}, L_{0}: X^{2} \rightarrow \mathbf{R}$ such that $f(x)=$ $a_{1}(x)+L_{1}(x, x)$ for $x \in X, h_{0}(x)-a_{0}(x)-L_{0}(x, x) \in \mathbf{Z}$ for $x \in X$, and $L_{0}(x, y)=L_{1}(x, y)=0$ whenever $x \perp y$. Next, according to Theorem 2.2 in [9] (see also Corollary 3.4 in [9]) and Proposition 11.1.1 in [1], there is a bilinear functional $L: X^{2} \rightarrow \mathbf{R}$ and $c_{1}, c_{0} \in \mathbf{R}$ with $L_{1}=c_{1} L$ and $L_{0}=c_{0} L$ (we use these theorem and proposition for functions $q_{i}: X \rightarrow \mathbf{R}, i=0,1$, given by: $q_{i}(x)=L_{i}(x, x)$ for $\left.x \in X\right)$. Putting $a_{2}=2 \pi i a_{0}$ and $c=c_{1}+2 \pi i c_{0}$ we obtain (25). The uniqueness of $a_{1}, a_{2}$, and $L$ results from the uniqueness of $a$ and $L$ in Theorem 2.11.

In the case where $X$ is a real linear topological space and $g$ is continuous at the origin the proof is analogous. It suffices only to use Corollary 2.10 instead of Theorem 2.11. This completes the proof 
Replacing, in the proof of Theorem 4.1, Theorem 2.11 by Corollary 2.14 we obtain the following.

Theorem 4.2. Let $(X, \perp)$ and $g$ be just the same as in Theorem 4.1. Then one of the following two conditions is valid.

(i) There are unique linear functionals $a_{1}, a_{2}: X \rightarrow \mathbf{R}$ with

$$
g(x)=\exp \left(a_{1}(x)+i a_{2}(x)\right) \quad \text { for } x \in X .
$$

(ii) There are $a \perp$-equivalent inner product $\langle\cdot, \cdot\rangle$ in $X, c \in \mathbf{C}$, and unique linear functionals $a_{1}, a_{2}: X \rightarrow \mathbf{R}$ such that

$$
g(x)=\exp \left(a_{1}(x)+i a_{2}(x)+c\|x\|^{2}\right) \quad \text { for } x \in X,
$$

where $\|x\|^{2}=\langle x, x\rangle$ for $x \in X$.

If, moreover, $X$ is a real topological linear space and $g$ is continuous at the origin, then $a_{1}$ and $a_{2}$ are continuous.

Taking into account Corollary 3.4 in [9], from Theorem 4.2 one can easily derive the following corollary (cf. Corollaries 1(i) and 2(i) in [3] and Corollary in [5]).

Corollary 4.3. Let $X$ be a real inner product space and $g: X \rightarrow \mathbf{C}$ be a hemicontinuous at the origin solution of (1). Then either $g(x)=0$ for every $x \in X$ or there exist $c \in \mathbf{C}$ and an $\mathbf{R}$-linear functional $a: X \rightarrow \mathbf{C}$ with $g(x)=\exp \left(a(x)+c\|x\|^{2}\right)$ for $x \in X$. If, moreover, $g$ is continuous at the origin, then a is continuous.

Finally we have the following.

Theorem 4.4. Let $(X, \perp)$ be an orthogonality space, $D \subset X$ be a set such that the origin is algebraically interior to $D$, and $g: X \rightarrow \mathbf{C}, g(X) \neq\{0\}$, be a solution of (1) which satisfies

$$
|g(x)|<\frac{4}{\sqrt{5}+1} \operatorname{Re}(g(x)) \quad \text { for } x \in D,
$$

where $\operatorname{Re}(z)$ denotes the real part of the complex number $z$. Then there exist an additive $a_{1}: X \rightarrow \mathbf{R}$, a linear $a_{2}: X \rightarrow \mathbf{R}$, a symmetric biadditive $L_{1}: X^{2} \rightarrow \mathbf{R}$, and a symmetric bilinear $L_{2}: X^{2} \rightarrow \mathbf{R}$ such that

$$
\begin{gathered}
L_{1}(x, y)=0=L_{2}(x, y) \quad \text { whenever } x \perp y, \\
g(x)=\exp \left(a_{1}(x)+L_{1}(x, x)+i\left(a_{2}(x)+L_{2}(x, x)\right)\right) \text { for } x \in X .
\end{gathered}
$$


Moreover, all these functionals are unique.

Proof. It is easily seen that, in the case where $g(x)=0$ for every $x \in X \backslash\{0\}$, condition (26) cannot be satisfied with any set $D \subset X$ such that the origin is algebraically interior to $D$. Thus, by Proposition $3.2,0 \notin g(X)$. Define the functions $f, h, h_{0}$, and $t$ in the same way as in the proof of Theorem 4.1. Then $f$ is orthogonally additive, $h_{0}$ satisfies (13) with $K=\mathbf{Z}$, and

$$
\frac{\sqrt{5}+1}{4}=\cos \frac{\pi}{5}<\operatorname{Re}(h(x)) \quad \text { for } \quad x \in D
$$

Thus, according to Corollary 7 in [13] and Proposition 11.1.1 in [1], there exist an additive functional $a_{1}: X \rightarrow \mathbf{R}$ and a biadditive symmetric functional $L_{1}: X^{2} \rightarrow \mathbf{R}$ such that

$$
f(x)=a_{1}(x)+L_{1}(x, x) \quad \text { for } \quad x \in X .
$$

Note that for every $x, y \in X$ with $x \perp y$ we have

$$
f(x)+f(y)=f(x+y)=a_{1}(x)+a_{1}(y)+L_{1}(x, x)+L_{1}(y, y)+2 L_{1}(x, y)
$$

and consequently $L_{1}(x, y)=0$. Further, (29) implies

$$
h_{0}(D) \subset \mathbf{Z}+\left(-\frac{1}{10}, \frac{1}{10}\right) .
$$

Hence, on account of Theorem 2.11, there exist a linear $a_{2}: X \rightarrow \mathbf{R}$ and a bilinear $L_{2}: X^{2} \rightarrow \mathbf{R}$ such that $L_{2}(x, y)=0$ whenever $x \perp y$ and

$$
h_{0}(x)-a_{2}(x)-L_{2}(x, x) \in \mathbf{Z} \quad \text { for } x \in X .
$$

This yields (27) and (28).

We show the uniqueness of $a_{1}$ and $L_{1}$ in a similar way as in the proof of Theorem 2.11; the uniqueness of $a_{2}$ and $L_{2}$ results directly from that theorem. This completes the proof.

Remark. The Remark on page 15 in [5] shows that the regularity assumptions, which we have made throughout the paper, are essential.

\section{References}

[1] J. Aczél and J. Dhombres, Functional equations in several variables, Encyclopedia of Mathematics and its Applications, Vol. 31, Cambridge University Press, 1989.

[2] K. Baron and G.L. Forti, Orthogonality and additivity modulo Z, Results Math., 26 (1994), 205-210. 
[3] K. Baron, F. Halter-Koch and P. Volkmann, On orthogonally exponential functions, Arch. Math. (Basel), 64 (1995), 410-414.

[4] K. Baron and PL. Kannappan, On the Pexider difference, Fund. Math., 134 (1990), 247-254.

[5] K. Baron and J. Rätz, Orthogonality and additivity modulo a subgroup, Aequationes Math., 46 (1993), 11-18.

[6] K. Baron and P. Volkmann, On the Cauchy equation modulo Z, Fund. Math., 131 (1988), 143-148.

[7] On a theorem of van der Corput, Abh. Math. Sem. Univ. Hamburg, 61 (1991), 189-195.

[8] L. Drewnowski and W. Orlicz, On orthogonally additive functionals, Bull. Acad. Polon. Sci. Sér. Sci. Math. Astronom. Phys., 16 (1968), 883-888.

[9] S. Gudder and D. Strawther, Orthogonally additive and orthogonally increasing functions on vector spaces, Pacific J. Math., 58 (1975), 427-436.

[10] R.C. James, Orthogonality and linear functionals in normed linear spaces, Trans. Amer. Math. Soc., 61 (1947), 265-292.

[11] Z. Kominek and M. Kuczma, Theorems of Bernstein-Doetsch, Piccard and Mehdi and semilinear topology, Arch. Math. (Basel), 52 (1989), 595-602.

[12] J. Lawrence, Orthogonality and additive functions on normed linear spaces, Coll. Math., 49 (1985), 253-255.

[13] J. Rätz, On orthogonally additive mappings, Aequationes Math., 28 (1985), 35-49.

[14] K. Sundaresan, Orthogonality and nonlinear functionals on Banach spaces, Proc. Amer. Math. Soc., 34 (1972), 187-190.

Received January 10, 1996.

Departament of Mathematics

Pedagogical University

REJTANA 16 A

35-310 Rzeszów, Poland 